\title{
Colorization by Landmark Pixels Extraction
}

\author{
Weiwei Du, Shiya Mori, and Nobuyuki Nakamori \\ Information Science, Kyoto Institute of Technology, \\ Kyoto, Japan 606-8585 \\ \{duweiwei, nakamori\}@kit.ac.jp, \\ m0651024@edu.kit.ac.jp
}

\begin{abstract}
A one-dimensional luminance scalar is replaced by a vector of a colorful multi-dimension for every pixel of a monochrome image, it is called as colorization. Obviously, it is under-constrained. Some prior knowledge is considered to be given to the monochrome image. Colorization using optimization algorithm is an effective algorithm for the above problem. Scribbles are considered as the prior knowledge. However, it cannot effectively do with complex images without repeating experiments for confirming the place of scribbles. Therefore, in our paper, landmark pixels are considered as the prior knowledge. We propose an algorithm which is colorization by landmark pixels extraction. It need not repeat experiments and automatically generates landmark pixels like scribbles. Finally, colorize the monochrome image according to requirements of user.
\end{abstract}

Keywords: Colorization, A monochrome image, Landmark pixels extraction.

\section{Introduction}

A one-dimensional luminance scalar is replaced by a vector of a colorful multidimension for every pixel of a monochrome image, it is called as colorization. Obviously, it is under-constrained. Consequently, there has no only one result to colorization. In order to solve above problem, we should give some priori knowledge or set a reasonable constraint.

Some prior knowledge is considered to be given to the monochrome image. There are representative local colorization algorithms such as Welsh [1] which is a semi-automatic colorization algorithm. It transfers colors referring a colorful image to the greyscale image. However, it is no guarantee that the continuity of the colors in space. There are representative global colorization algorithms such as Levin [2] which is a colorization using optimization algorithm. Its basic idea: neighboring pixels in space and time that have similar intensities should have similar colors. User must draw some color scribbles to the monochrome image as some prior knowledge(Fig.3(a)). The indicated colors are propagated in both space and time to produce a fully colorized image. It may colorize the monochrome image in the context of not segmenting it to regions directly. It is an effective algorithm for a simple monochrome image. 
However, Levin's method cannot effectively colorize monochrome images such as Fig.4(a) which is given as some scribbles at random by user. If we want to get the result of Fig3.(b), Levin's method must be repeated experiments for confirming the place of scribbles with Fig.4(a). It becomes a key point how to get some prior knowledge automatically. [5] obtained the distance of colors by repeating [2]'s method for extracting landmark pixels, while we obtained the distance of luminance by classfication for extracting landmark pixels. Therefore, in our paper, it need not repeat experiments and automatically generates landmark pixels like scribbles. Landmark pixels are considered as the prior knowledge. We give some colors landmark pixels. Finally, colorize the monochrome image based on colorful landmark pixels according to requirements of user.

\section{Algorithm}

\subsection{Generate Landmark Pixels}

Landmark pixels are some representative pixels in an image. It costs much time if landmark pixels are extracted from an original image directly. Hence, degrade the monochrome image to low resolution image. The initial landmark pixels are extracted from the low resolution image using k-means. And then upgrade the resolution image, at the same time, we raise the number of landmark pixels. We repeat the above process until the result is the same as the resolution of the original image.

A monochrome image $I_{0}$ is given. We build a gaussian pyramid $I_{0}, I_{1}, \ldots, I_{d}$, where $I_{0}$ is the input monochrome image of the original image and $I_{d}$ is the coarsest level in the pyramid. We classify the coarsest level image $I_{d}$ using information on the value of each pixel and position of each pixel. $\mathrm{K}$ clusters are obtained using k-means. The centroid of each cluster is considered as the initial landmark pixels. Let set of the initial landmark pixels be $X_{d}$. The mean value is substituted for the values of all pixels of each cluster. Let the image be $\Phi_{d}$. The residue image is obtained by eq.(1) when $i=d$.

$$
E_{i}=\left|I_{i}-\Phi_{i}\right|
$$

We divide $E_{d}$ into small windows like Fig.1. The size of each window is $h \times h$ pixels. Suppose the size of the input image is $M \times N$ pixels. We build a gaussian pyramid with a scale factor 2 . Suppose that we set one landmark pixel to a window in the coarsest level image. $c$ is the number of the landmark pixels. We will add at most $\frac{M}{2^{k} h} \times \frac{N}{2^{k} h}$ landmark pixels to small windows. Based on the idea, we can get $h$ by eq.(2) and eq.(3). We set the same threshold to every small window. The value of the pixel should be memorized, if the mean value of pixel of the small window is larger than the threshold. The threshold is set at 30 based on experiments, if the number of landmark pixels is larger than 300 . Otherwise, the threshold is set at 20. It is difficult to get the landmark pixels, if the threshold is too large. Otherwise, it will cost much time in order to extract many landmark pixels. 


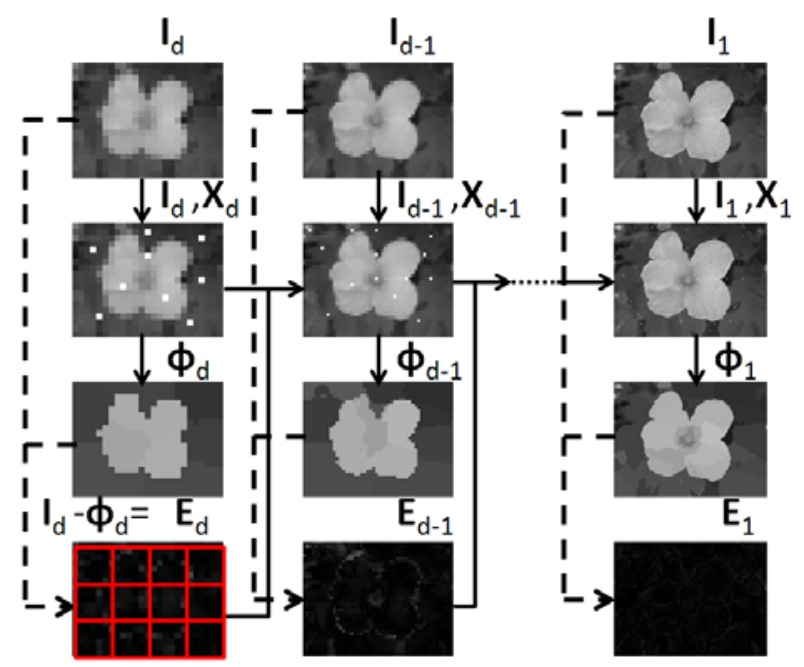

Fig. 1. Flowchart on process of generating landmark pixels

$$
\begin{gathered}
\sum_{k=0}^{d} \frac{M}{2^{k} h} \times \frac{N}{2^{k} h}=c \\
h=\sqrt{\frac{4 M N}{3 c}\left(1-\frac{1}{4^{d+1}}\right)}
\end{gathered}
$$

We can get the landmark pixels $X_{d-1}$ from image $E_{d}, \Phi_{d-1}$ is obtained by segmenting $I_{d-1}$ based on the set $X_{d-1}$ of landmark pixels by K nearest neighbor. The residue image $E_{d-1}$ can be obtained by eq.(1), when $k=d-1$. In this way, until the landmark pixels $X_{0}$ are extracted from the image $I_{0}$.

\subsection{Classify Landmark Pixels}

We should give every landmark pixel colorize the corresponding color. However, many landmark pixels are extracted from a monochrome image so that we are not able to colorize every landmark pixel. Fortunately, we find some landmark pixels should be set the same color. The same color landmark pixels would be colorized, if we only colorize a landmark pixel of the same color landmark pixels. According to this idea, we classify the landmark pixels to some clusters using ward's method. That is, the clusters of similarity have the small sum of squares while the clusters of difference have the large sum of squares in ward's method. We make the landmark pixels of the same cluster have the same color. We just colorize a landmark pixel of the same cluster, all landmark pixels obtain the same color in the cluster. 


\subsection{Colorize Landmark Pixels}

How to colorize the monochrome image using the colored landmark pixels. We adopt Levin's method as her algorithm requires neither precise image segmentation, nor accurate region tracking. The basic idea of the algorithm: if neighboring pixels in space and time have similar intensities, they should have similar colors. That is to say, when the monochromatic luminance channel Y are similar, the chrominance channels $\mathrm{U}$ and $\mathrm{V}$ are similar. YUV color space is used in video 3 . In a word, it is a process to solve the solution of a quadratic cost function in sparse system of linear equations. The color scribbles are conditions of constraints in order to solve problem. In our paper, the color landmarks substitute for the color scribbles as conditions of constraints. The color landmarks more effective than the color scribbles without repeating experiments for confirming the place of scribbles.

\subsection{Steps of Our Algorithm}

Fig.2 shows the process of algorithm. It is carried out according to the following procedure.

1. Degrade an image to the low resolution image with downsampling.

2. Classify the low resolution image for initial landmark pixels.

3. Substitute the mean value of each cluster for the values of all pixels and obtain the image $\Phi$.

4. Obtain the residue image $\mathrm{E}$ by $\|I-\Phi\|$.

5. Segment the residue image $\mathrm{E}$ with small windows so that the landmark pixels are added with these windows.

6. Classify the landmark pixels by ward's method.

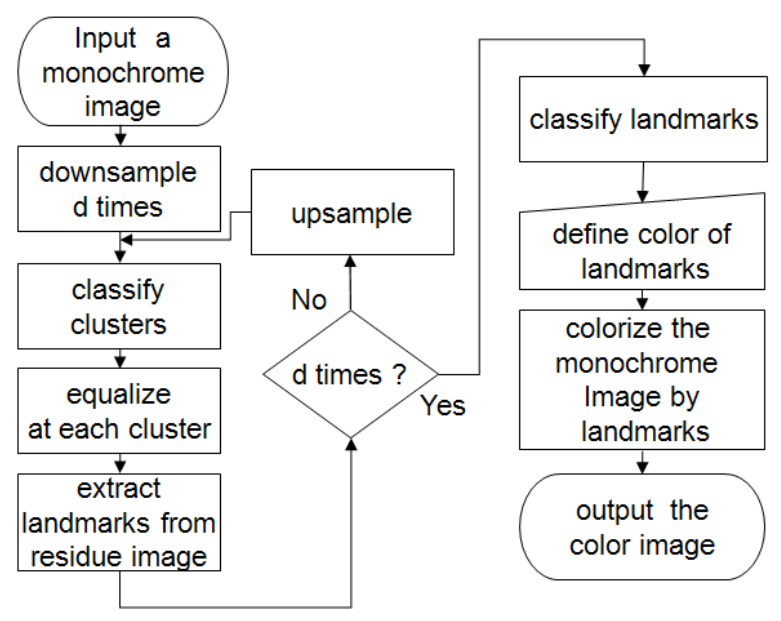

Fig. 2. Flowchart on process of our proposal 
7. Define the landmark pixels of the same cluster to the same color.

8. Colorize the monochrome image by the defined colored lardmark pixels. Repeat from step 3 to step 5 until obtain the original image. After that, go ahead to step 6 until obtain a color image.

\section{Experiments}

Fig.3(a) and Fig.6(a) show the monochrome image with scribbles and its result with colorization from [2. We draw some colored scribbles to the monochrome image freely like Fig.4(a) and Fig.7(a). We cannot obtain the result such as Fig.3(b) and Fig.6(b) while obtain Fig.4(b) and Fig.7(b). So we know it is not easy to get the result like Fig.3(b) and Fig.6(b). Experiments should be done until the result like Fig.3(b) and Fig.6(b) is obtained. Only by appropriately drawing the colored scribbles, Fig.3(b) and Fig.6(b) can be obtained. Our proposal does not consider the above problem for comparison. Our algorithm can generate landmark pixels automatically like Fig.5(a) and Fig.8(a). We just colorize one landmark pixel of each cluster and then can obtain the result like

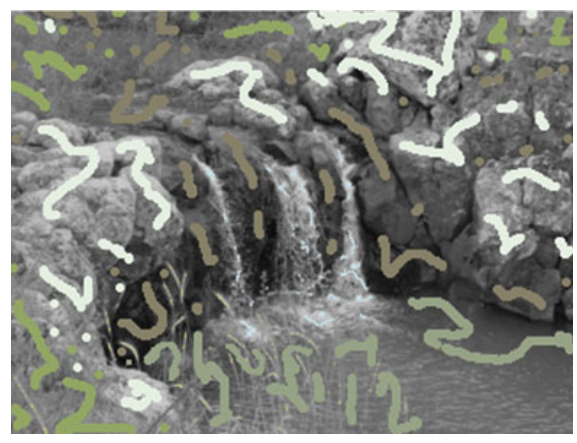

Fig. 3(a). The monochrome waterfall image with scribbled colors

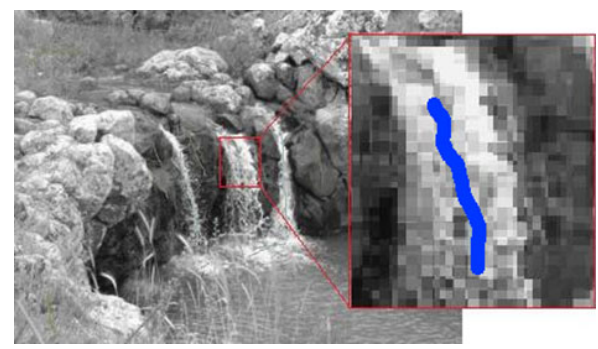

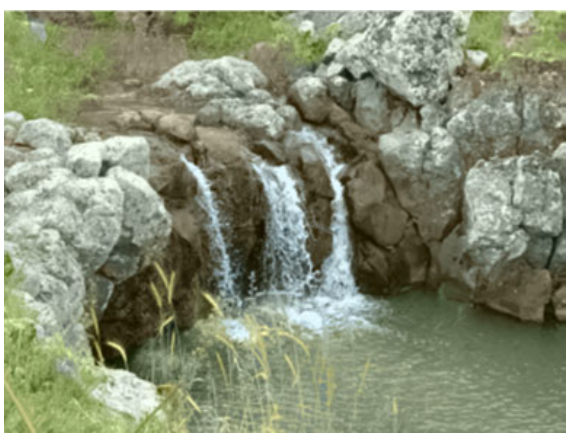

Fig. 3(b). The result of the color waterfall image

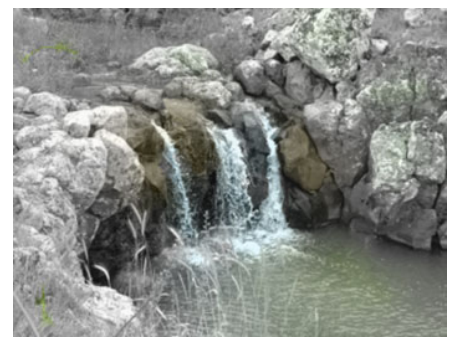

Fig. 4(a). The monochrome waterfall im- Fig. 4(b). The result of the color waterfall age with scribbled colors image 


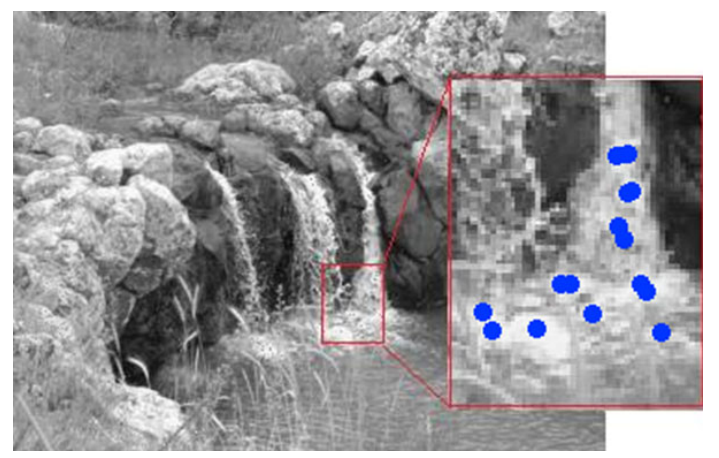

Fig. 5(a). The monochrome waterfall image with landmark pixels

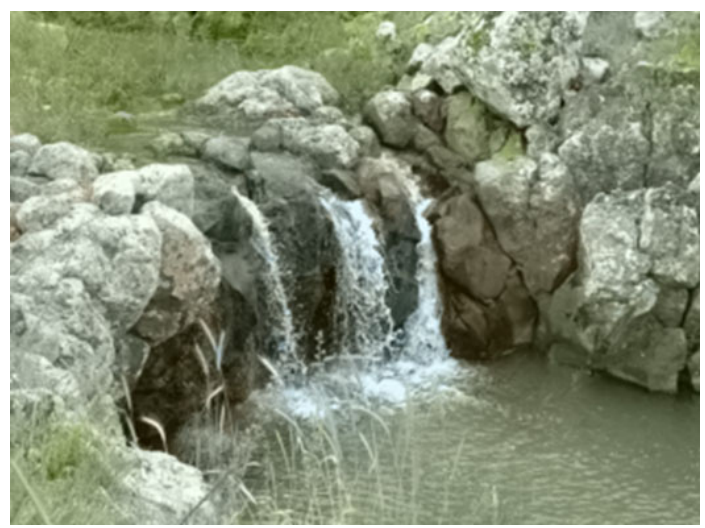

Fig. 5(b). The result of the color waterfall image

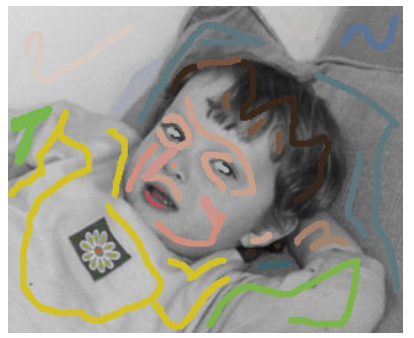

Fig. 6(a). The monochrome child image with scribbled colors

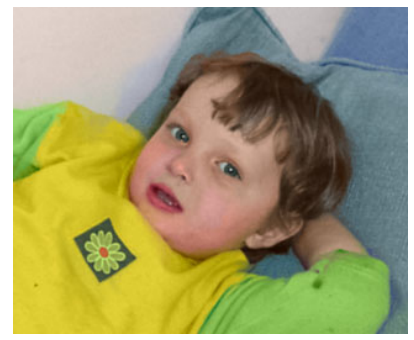

Fig. 6(b). The result of the color child image 


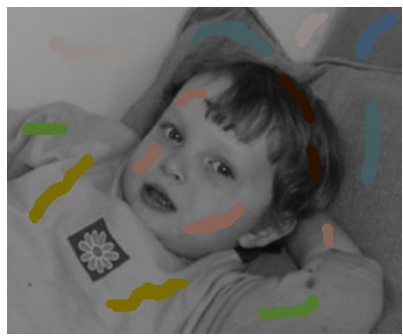

Fig. 7(a). The monochrome child image with scribbled colors

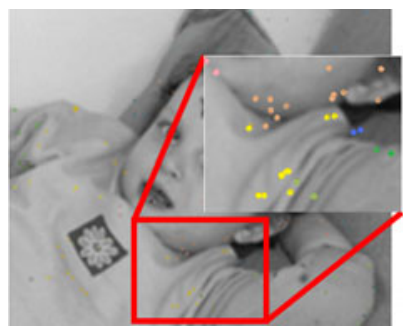

Fig. 8(a). The monochrome child image with landmark pixels

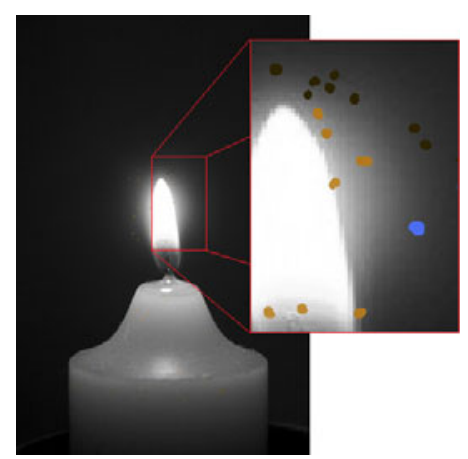

Fig. 9(a). The monochrome candle image with landmark pixels

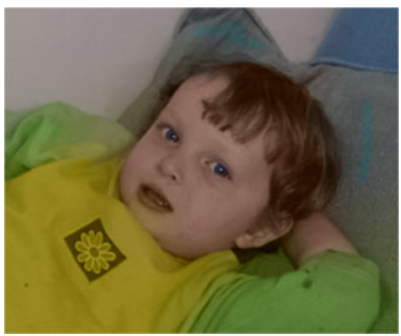

Fig. 7(b). The result of the color child image

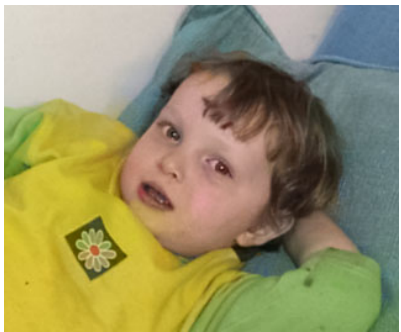

Fig. 8(b). The result of the color child image

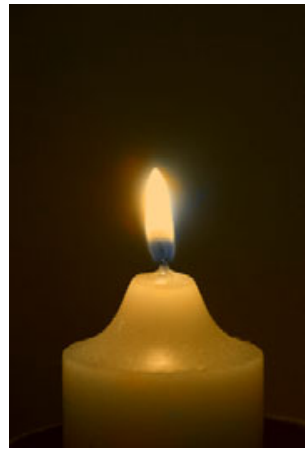

Fig. 9(b). The result of the color candle image

Fig.5(b) and Fig.8(b). Some parameters of our proposal are given on Fig.5(b): the number of landmark pixels is $c=700$, threshold is $T=20$, the number of levels is $d=4$, the size of a small window is $h=13$, the number of clusters is $n=25$. On Fig.8(b): the number of landmark pixels is $c=300$, threshold is $T=20$, the number of levels is $d=5$, the size of a small window is $h=20$, the number of clusters is $n=100$. We carried out some experiments to other images. Their results are showed at Fig.9(b) and Fig.10(b). 


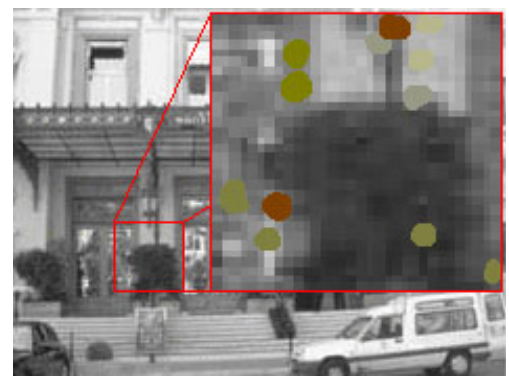

Fig. 10(a). The monochrome building image with landmark pixels

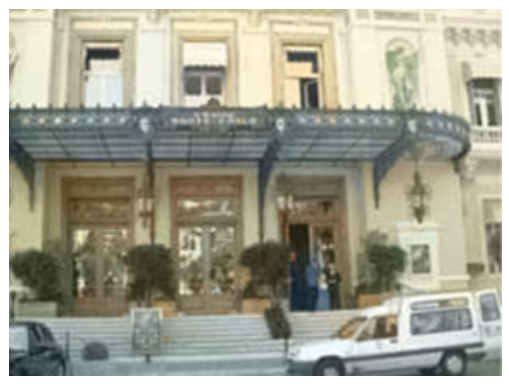

Fig. 10(b). The result of the color building image

\section{Conclusion}

In this paper, we present an effective colorization method using landmark pixels. Automatically generating landmark pixels is the advantage of the algorithm. However, we must define a color in the one landmark pixel of each cluster manually. Therefore, automatically defining a color in the one landmark pixel of each cluster is the subject of future research.

\section{References}

1. Welsh, T., Ashikhimin, M., Mueller, K.: Transferring color to greyscale images. ACM Transactions on Graphics 21(3), 277-280 (2002)

2. Levin, A., Lischinski, D., Weiss, Y.: MVA Conference: Colorization using optimization. In: Proceedings of ACM SIGGRAPH 2004, pp. 689-694 (2004)

3. Jack, K.: Video demystified, 3rd edn. Elsevier Science and Technology (2001)

4. Ward, J.H.: Hierarchical grouping to optimize an objective function. Journal of the American Statistical Association 58, 236-244 (1963)

5. Huang, T.W., Chen, H.T.: Landmark-Based Sparse Color Representation for Color Transfer. In: The 12th IEEE International Conference on Computer Vision, pp.199$204(2009)$ 